\title{
Prehospital Diagnosis and Management of Acute Myocardial Infarction
}

\author{
Adam J. Brown, Francis J. Ha, Michael Michail, \\ and Nick E. J. West
}

\section{$2.1 \quad$ Introduction}

The outcomes of patients suffering from acute myocardial infarction are contingent on the time taken to deliver definitive treatment. Evidence has shown that the extent of myocardial salvage is greatest if patients are reperfused in the first $3 \mathrm{~h}$ from the onset of symptoms [1]. For every 30-min delay in coronary reperfusion, the relative 1 -year mortality rate increases by $7.5 \%$ [2]. This has driven physicians and policymakers to popularize phrases such as 'time is myocardium' and concepts such as 'door-to-balloon' time- the latter representing the time to reperfusion with an intracoronary device from the arrival of the patient at hospital (Fig. 2.1). The biggest delays and challenges in reducing the time to reperfusion, however, are in fact mostly seen in the prehospital setting. This consists of the time from the onset of symptoms to first medical contact (FMC) and subsequently the time from FMC to diagnosis and then reperfusion treatment—-termed 'system delay'. Patient delay may be multifactorial and depends on a host of issues including socioeconomic factors and access to healthcare. The rapid patient assessment and field diagnosis of myocardial infarction has become a crucial factor in time to reperfusion as it dictates the decision on the most appropriate form of reperfusion treatment accounting for geographical factors and available facilities. Importantly, the prehospital role in

\footnotetext{
A. J. Brown $(\bowtie) \cdot$ F. J. Ha $\cdot$ M. Michail

MonashHeart, Monash Health and Monash Cardiovascular Research Centre, Monash University, Clayton, VIC, Australia

Department of Interventional Cardiology, Papworth Hospital, Cambridge, UK e-mail: ajdbrown@me.com

N. E. J. West

Department of Interventional Cardiology, Papworth Hospital, Cambridge, UK
} 


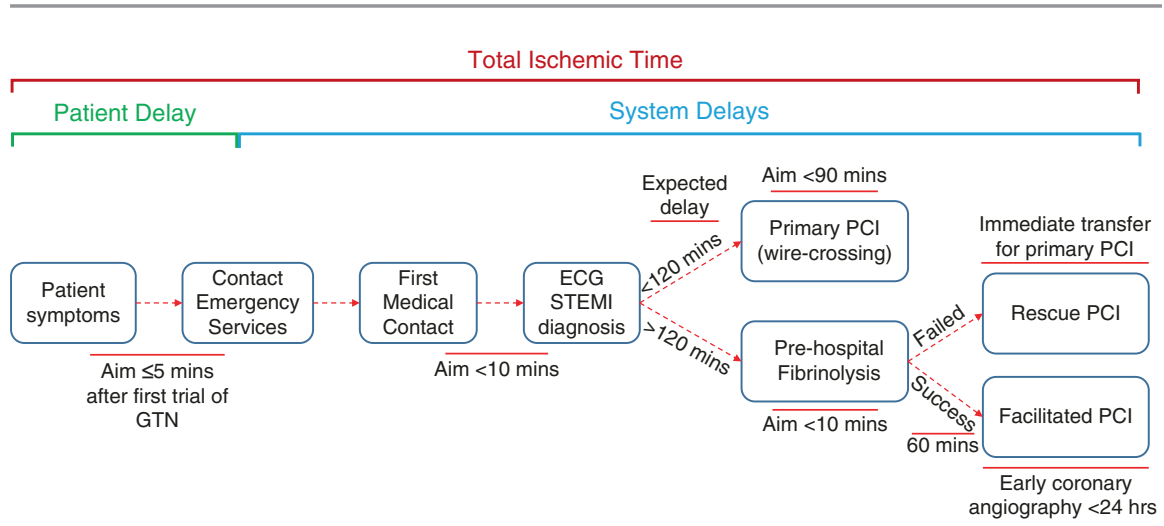

Fig. 2.1 The key components of symptom to revascularization time indicating recommended timing for optimal outcomes. Note that optimization of each component offers an opportunity to minimize total ischaemic time

the management of acute myocardial infarction also involves the initiation of therapy, the upstream of the hospital-delivered treatment. This commonly involves the administration of antiplatelet and anticoagulant therapy in metropolitan areas, while in remote areas where patients cannot be transferred to hospital facilities in a reasonable time, there are policies in place for administration of field thrombolytic agents. Other aspects of the management may involve intravenous access or, indeed in the cases of cardiac arrest, cardiopulmonary resuscitation. In this chapter we will discuss the management of patients presenting with acute myocardial infarction, in the crucial period ahead of their arrival at the heart attack centre.

\subsection{Activation of Emergency Services}

\subsubsection{The Call for Help}

The diagnosis and treatment of acute myocardial infarction (MI) in the community is dependent on the recognition of clinical symptoms. Awareness of potential warning signs is largely dependent on the patient, yet it is not uncommon for patients to wait $2 \mathrm{~h}$ or more after symptom onset before contacting emergency medical services (EMS) [3]. Reasons for delayed presentation include perception of the 'Hollywood heart attack' (despite up to one-third of patients presenting without angina), differing symptoms compared with previous experiences in patients with known ischaemic heart disease, fear of embarrassment or troubling others and persistent attempts at self-medication. Thus, community awareness and patient education remain a cornerstone of early diagnosis. Public health initiatives encompass identification of key warning signs such as chest discomfort radiating to relevant areas, light headedness or dyspnoea, as well as advocacy of contacting EMS within 5 min of symptom onset. Furthermore, targeted health campaigns towards higher-risk 
individuals such as those with relevant cardiovascular comorbidities, alongside their families, are a fundamental aspect of primary and secondary prevention. However, systemic factors such as access to EMS, provision of health insurance and socioeconomic background also influence the time to presentation, and policy-makers hold a substantial role in determining patient outcomes.

Following symptom recognition, prompt contact with EMS is necessary. Where previously prescribed, patients may be given a trial of nitroglycerine; however, the worsening or persistence of symptoms (usually beyond $5 \mathrm{~min}$ after administration) is indicative of non-response and mandates EMS contact. There is also the risk of systemic hypotension after repeated nitroglycerin administration which may precipitate cardiogenic shock in the context of acute myocardial infarction. Private transportation as opposed to EMS transfer is unsafe given the risk of cardiac arrest in the absence of any trained medical support. Other benefits of prompt EMS contact include the opportunity to perform an ECG leading to earlier diagnosis, subsequent assessment of timely transfer to primary PCI centre and early fibrinolytic therapy by adequately trained healthcare providers.

\subsubsection{First Medical Contact}

First medical contact (FMC) is the time at which trained EMS providers who can obtain and interpret the ECG arrive at the patient's side. The task faced by EMS providers upon initial patient contact should not be underestimated. An assessment of the patient's need for immediate life support, attainment of key history from the patient and witnessing bystanders and focused clinical examination must be performed efficiently and accurately for appropriate triage. This evaluation may be complicated by atypical but not uncommon presentations such as those in elderly, female, diabetic or cognitively impaired patients. The availability of a defibrillator is a mandatory part of EMS given the initial presentation may be cardiac arrest. Subsequently, a prehospital 12- or 18-lead ECG is crucial in the context of relevant symptoms and can be electronically transmitted to a hospital doctor or interpreted by EMS personnel with adequate training. A prehospital diagnosis of STEMI combined with direct referral to a primary PCI centre reduces time to device intervention and all-cause mortality [4]. In brief, significant ST-segment changes or left bundle branch block with sufficient clinical suspicion warrants exclusion of acute myocardial infarction. At this point and ideally less than 10 min from FMC, EMS providers should activate the 'STEMI pathway' in which patients may be transferred to a centre capable of primary PCI, a non-PCI centre for fibrinolysis, or receive prehospital fibrinolysis during transfer. While immediate transfer to a primary PCI centre is ideal, this is largely dependent on the presence of an established STEMI activation pathway, a coordinated hospital network and regional geography. Moreover, local protocols that facilitate prehospital registration may be in place to transfer patients directly to the catheterization lab, effectively bypassing the emergency department and further reducing unnecessary delays. Current guidelines recommend a FMC to device intervention time of 120 min or less when considering 
Fig. 2.2 Mortality reduction as a function of total ischaemic time. Note that extent of myocardial salvage dramatically reduces within the first $4 \mathrm{~h}$ but that up to $24 \mathrm{~h}$, the salvage of a smaller proportion of myocardium is still possible

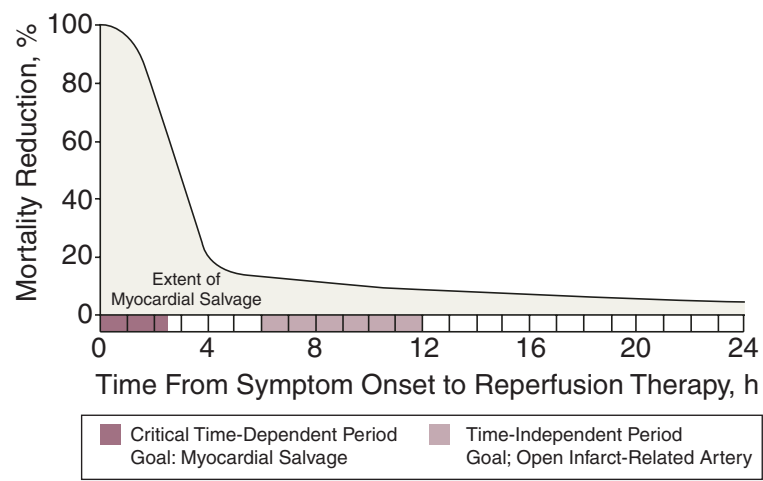

primary PCI [5]; however, any system must ultimately focus on reducing total ischaemic time irrespective of the mode of reperfusion therapy (Fig. 2.2). Caution should be exercised concerning overdependence on primary PCI where facilities may not be within reasonable accessibility.

\subsection{Diagnosis of Myocardial Infarction}

The diagnosis of an acute myocardial infarction is made when a patient has elevated blood serum levels of cardiac enzymes (preferably cardiac troponin) and one or more of the following: (a) symptoms suggestive of myocardial ischaemia, (b) ECG demonstrating new significant ST-T changes or new left bundle branch block (LBBB), (c) new pathological Q waves on ECG, (d) imaging evidence of new loss of viable myocardium or new regional wall motion abnormality or finally (e) identification of intracoronary thrombus on angiography or autopsy [6]. The suspicion of myocardial infarction usually begins from the point at which a call is made to the emergency medical services (EMS). At the point of FMC, a working diagnosis of STEMI must be made as soon as possible, and therefore a focused history and 12-lead electrocardiogram must be performed with a maximum target delay of 10 min. The clinical history is a critical component of the diagnosis of STEMI, with particular emphasis on the nature of the chest pain. While other mimics of STEMI such as myopericarditis or aortic dissection would be important to consider at an early stage as they would affect the subsequent management, acute myocardial infarction should remain at the forefront of the differential diagnoses as it remains common and is time critical in its management.

\subsubsection{History and Examination}

A focused history and examination must be carried out promptly at FMC. Symptoms suggestive of myocardial ischaemia may include chest pain which can radiate to the left arm, neck and/or jaw. Chest pain may be associated with shortness of breath, 
diaphoresis, nausea, vomiting, palpitations or even syncope. The early recognition of patients with decompensated heart failure may enable the early administration of diuretics. Pulse rate, blood pressure monitoring and oxygen pulse oximetry should be monitored. It is recommended that as soon as the suspicion of a diagnosis of acute myocardial infarction is made, that ECG monitoring (with defibrillator capacity) is carried out as these patients are at high risk of malignant arrhythmias.

\subsubsection{ECG Diagnosis}

The 12-lead ECG should be calibrated at $10 \mathrm{~mm} / \mathrm{mV}$ with a standard paper (or screen) speed of $25 \mathrm{~mm} / \mathrm{s}$. The interpretation of the ECG should be made by a trained medical professional; otherwise, field transmission of the ECG should be done for immediate diagnosis. An ECG diagnosis of STEMI is made with new ST-segment elevation at the J point in two contiguous leads at (a) $\geq 0.2 \mathrm{mV}$ in $\geq 40$ years, $\geq 0.25 \mathrm{mV}$ in men $<40$ years or $\geq 0.15 \mathrm{mV}$ in women in leads $V_{2}-V_{3}$ and/ or (b) $\geq 0.1 \mathrm{mV}$ in all other leads (Fig. 2.3). The presence of reciprocal ST depression helps confirm the diagnosis and differentiates myocardial ischaemia from other causes of ST-segment abnormalities, such as left ventricular aneurysm or even noncardiac causes such as subarachnoid haemorrhage. New LBBB in the context of a highly suggestive history should be treated as acute myocardial infarction. While LBBB is not specific for an MI, one which is related to an infarct is more likely to be inferred a larger infarct size with poorer prognosis. Associated features such as decompensated cardiac failure and cardiogenic shock should raise clinical suspicion [7]. While various algorithms have been proposed to aid the diagnosis in the context of LBBB, they do not offer sufficient diagnostic certainty. Findings in the

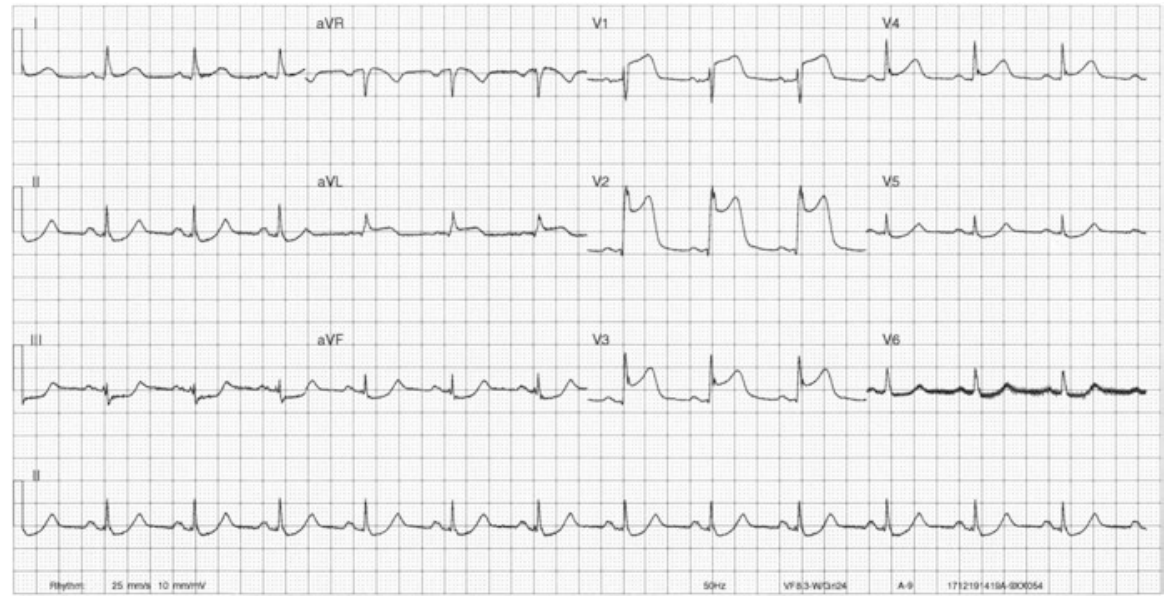

Fig. 2.3 Anterior ST-segment-elevation ECG. More marked ST-segment elevation $V_{1}-V_{4}$ with reciprocal change in other leads 
Table 2.1 Localizing of myocardial infarction on ECG

\begin{tabular}{l|l|l}
\hline Infarct location & ECG changes & Affected coronary artery \\
\hline Septal & $V_{1}-V_{2}$ & Septal LAD \\
\hline Anterior & $V_{3}-V_{4}$ & LAD \\
\hline Lateral & $\mathrm{I}$, aVL $, V_{5}, V_{6}$ & LCx, diagonals \\
\hline Inferior & II, III, aVF & LCx $(15 \%)$, RCA $(85 \%)$ \\
\hline Posterior & $V_{7}, V_{8}, V_{9}$ (posteriorly placed) & RCA \\
\hline
\end{tabular}

context of LBBB which should raise clinical suspicion include (a) concordant ST-segment elevation $\geq 1 \mathrm{~mm}$ in leads with positive QRS complex, (b) concordant ST-segment depression $\geq 1 \mathrm{~mm}$ in $V_{1}-V_{3}$ and (c) discordant ST-segment elevation $\geq 5 \mathrm{~mm}$ in leads with a negative QRS complex. Given that right bundle branch block (RBBB) in the context of a STEMI often has ambiguous ST segments and has also been associated with a poor prognosis, recent guidelines have advised a change in approach in the management of such patients. In the context of persistent ischaemic symptoms, they should be treated in a similar fashion to LBBB with emergent coronary angiography and PCI if indicated. Likewise, patients with permanent pacemaker devices that are set up for right ventricular pacing will exhibit ECG changes similar to that of left bundle branch block. Of note, a patient with an ECG demonstrating ST-segment elevation in aVR and/or $V_{1}$ with otherwise widespread ST-segment depression should raise the suspicion of left main coronary artery obstruction, particularly if coupled with haemodynamic compromise. The localization of myocardial infarction can be also be deduced from the ECG as in table (Table 2.1).

While those with STEMI are at greatest risk from morbidity and mortality, patients presenting with non-ST elevation acute coronary syndromes (NSTEACS) also benefit from angiography and revascularization for prognostic advantage. It has become evident however that patients with NSTEACS and high-risk features benefit from early intervention, and as such, guidelines are now advocating angiography for this group within $24 \mathrm{~h}$ of presentation [8,9]. In order to deliver this high standard of care, select ambulance services have recently adopted a direct access pathway which facilitates patients with certain high-risk clinical and ECG features to be brought directly to a heart attack centre. These protocols frequently include patients with (a) persistent ST depression $>1 \mathrm{~mm}$ or transient ST elevation, (b) pathological T-wave inversion in $V_{1}-V_{4}$, (c) dynamic T-wave inversion $>2 \mathrm{~mm}$ in two or more contiguous leads or (d) haemodynamic (e.g. sustained hypotension $>15 \mathrm{~min}$, pulmonary oedema, heart failure) or electrical (e.g. sustained ventricular tachycardia or fibrillation) instability thought to be secondary to cardiac ischaemia.

\subsubsection{Cardiac Biomarkers}

Point-of-care cardiac biomarkers are well established and demonstrate excellent diagnostic performance compared with lab-based assays. Their use in the 
prehospital setting is still being evaluated, but use of these tests may provide some guidance in the appropriate triaging of patients with suspected acute myocardial infarction, especially in cases where there is diagnostic uncertainty, such as ambiguous chest pain or in the unconscious patient. Some cardiologists have raised concerns that serum levels of cardiac biomarkers may be undetectable or below reference range if they are measured very early in an evolving myocardial infarction, which may in turn provide false reassurance to the practitioner assessing the patient. Their use has therefore been limited to few ambulance services, and their benefit to patient care will continue to be appraised.

\subsection{Prehospital Treatment of Myocardial Infarction}

\subsubsection{Principles of Prehospital Therapy}

Prehospital therapy for acute MI focuses on reducing thrombus burden and coagulation cascade hyperactivity prior to coronary reperfusion treatment. Potential therapies include antiplatelets, anticoagulants and glycoprotein (GP) IIb/IIIa inhibitors; the combination in which they are used is often institution or even operator dependent (Table 2.2). The evidence to support each type of therapy and specific drugs within each class varies, although current class 1 guideline recommendations are prompt dual antiplatelet therapy in the form of aspirin and a potent $\mathrm{P} 2 \mathrm{Y}_{12}$ inhibitor and an appropriate anticoagulant after assessment of bleeding risk [5]. Total ischaemic time remains the priority, and administration of these adjunct agents should not delay EMS transfer. While the optimal coronary reperfusion is primary PCI, such facilities may not be readily available to all patients. In such cases, prehospital fibrinolytic therapy can be utilized for initial reperfusion.

\subsubsection{Antiplatelet Therapy}

The benefits of dual antiplatelet therapy (DAPT) in patients undergoing primary PCI are well established. While initially borne out in the context of reducing the ongoing and persistent risk of stent thrombosis, DAPT also reduces the risk of subsequent spontaneous MI in non-stented coronary segments and all-cause mortality [10]. In the prehospital setting, aspirin should be administered as soon as possible. The dissolved or chewed oral form is preferable over swallowing a whole tablet due to more rapid absorption, with a recommended dose of $150-300 \mathrm{mg}$. There is a paucity of data evaluating intravenous aspirin therapy in the setting of STEMI although given its 50\% oral bioavailability, a corresponding dose between 75 and $150 \mathrm{mg}$ is appropriate in patients unable to tolerate the oral route. In contrast, the timing for initiation of adjunct $\mathrm{P}_{2} \mathrm{Y}_{12}$ inhibition therapy, including ticagrelor, prasugrel or clopidogrel, is uncertain. The ATLANTIC trial aimed to evaluate the safety, ECG changes and pharmacodynamics based on timing of $\mathrm{P}_{2} \mathrm{Y}_{12}$ inhibition therapy in the form of ticagrelor in early-presenting STEMI patients [11]. In 1862 patients 
Table 2.2 Adjuvant pharmacotherapies used in the prehospital setting

\begin{tabular}{|c|c|c|c|}
\hline $\begin{array}{l}\text { Adjunct } \\
\text { pharmacologic } \\
\text { therapies }\end{array}$ & $\begin{array}{l}\text { Recommendations } \\
\text { in primary PCI }\end{array}$ & Evidence & Notes \\
\hline Aspirin & +++ & $\begin{array}{l}\text { ISIS-2, } \\
\text { collaborative } \\
\text { meta-analysis }\end{array}$ & $\begin{array}{l}\text { - Dissolved or chewed } \\
\text { - Loading dose }(\geq 150 \mathrm{mg})\end{array}$ \\
\hline \multicolumn{4}{|l|}{$P 2 Y_{12}$ inhibitors } \\
\hline Clopidogrel & ++ & $\begin{array}{l}\text { COMMIT, } \\
\text { CURE }\end{array}$ & - Slower onset vs. prasugrel/ticagrelor \\
\hline Prasugrel & +++ & $\begin{array}{l}\text { Triton-TIMI 38, } \\
\text { ETAMI }\end{array}$ & $\begin{array}{l}\text { - Avoid in patients with previous stroke/ } \\
\text { TIA or high risk of bleeding (previous } \\
\text { haemorrhagic stroke, oral } \\
\text { anticoagulants, liver disease) }\end{array}$ \\
\hline Ticagrelor & +++ & PLATO & $\begin{array}{l}\text { - Avoid in patients at high risk of } \\
\text { bleeding }\end{array}$ \\
\hline Cangrelor & ++ & $\begin{array}{l}\text { CHAMPION } \\
\text { PHOENIX }\end{array}$ & $\begin{array}{l}\text { - IV administration } \\
\text { - Rapid onset and offset }\end{array}$ \\
\hline \multicolumn{4}{|l|}{ Anticoagulants } \\
\hline UFH & +++ & $\begin{array}{l}\text { (Operator } \\
\text { familiarity) }\end{array}$ & $\begin{array}{l}\text { - Can measure anticoagulation with } \\
\text { ACT }\end{array}$ \\
\hline Enoxaparin & +++ & ATOLL & - Better clinical outcomes than UFH \\
\hline Bivalirudin & ++ & MATRIX & $\begin{array}{l}\text { - More expensive than heparin } \\
\text { - Consider in patients with HIT }\end{array}$ \\
\hline Fondaparinux & - & OASIS 6 & $\begin{array}{l}\text { - No demonstrated benefit in primary } \\
\text { PCI }\end{array}$ \\
\hline \multicolumn{4}{|c|}{ GP IIb/IIIa inhibitors } \\
\hline Abciximab & + & FINESSE & \multirow{2}{*}{$\begin{array}{l}\text { - Consider as bailout therapy in } \\
\text { angiographic evidence of no or slow } \\
\text { reflow or large thrombus }\end{array}$} \\
\hline Tirofiban & + & On-TIME 2 & \\
\hline
\end{tabular}

Recommendations range from '+++' strongly recommended to '-' not recommended

randomized to prehospital or in-hospital ticagrelor with a median time difference between strategies of $31 \mathrm{~min}$, there was no between-group difference in the co-primary end point of pre-PCI resolution of ST-segment elevation or absence of TIMI flow grade 3 in infarct-related artery on angiography. However, there was a trend favouring prehospital ticagrelor for the resolution of ST-segment elevation after PCI which is consistent with the pharmacokinetic data of drug onset time. As such, the current practice is prompt prehospital DAPT in STEMI patients undergoing primary PCI. Prasugrel and ticagrelor are superior to clopidogrel in patients with acute coronary syndrome (ACS) across a range of cardiovascular outcomes including recurrent MI and stent thrombosis, while also having a faster onset of action [12-14]. An initial loading dose is needed to rapidly attain a therapeutic concentration; however, patients should be assessed for bleeding risk, exclusion of other possible diagnoses and drug-specific contraindications before administration. Cangrelor is another $\mathrm{P} 2 \mathrm{Y}_{12}$ inhibitor administered intravenously with benefits including rapid onset and superiority over clopidogrel for the composite end point of death, MI, 
ischaemic-driven revascularization or stent thrombosis in patients undergoing PCI [15]. It has yet to be compared with prasugrel or ticagrelor and its use limited to patients yet to receive oral $\mathrm{P} 2 \mathrm{Y}_{12}$ inhibition therapy.

\subsubsection{Anticoagulants}

The use of prehospital anticoagulant therapy for primary PCI has been mostly derived from extensive experience and familiarity rather than established clinical evidence. Despite the lack of a placebo-controlled trial for anticoagulants in primary PCI, they are still frequently used in clinical practice. Unfractionated heparin (UFH) is the standard anticoagulant for primary PCI. Enoxaparin is an alternative and has been associated with a reduction in composite risk of death, recurrent ACS or urgent revascularization at 30 days (7\% vs. $11 \%$, respectively; $p=0.02)$ compared with UFH in the ATOLL trial [16]. However, the ability to measure UFH anticoagulation through activated clotting time (ACT) has led to UFH remaining the standard of care for PCI. Bivalirudin may be considered with the MATRIX trial reporting lower mortality and bleeding at the expense of increased absolute stent thrombosis events compared with UFH in 7213 ACS patients [17]. Its comparatively higher cost and lack of prehospital data have limited its use, although it may be preferred in patients at greater risk of bleeding, especially when infused for longer durations ( $>4 \mathrm{~h}$ ) post-PCI. Conversely, fondaparinux has shown no benefit in primary $\mathrm{PCI}$ and is not recommended in this setting. However it may be an attractive alternative in STEMI patients not suitable for primary PCI with reduced death and re-infarction compared with UFH at 3-6 months (hazard ratio $0.77,95 \%$ CI 0.64 $0.93, p=0.008$ ) shown in the OASIS-6 trial [18].

\subsubsection{GP IIb/IIIa Inhibitors}

Prehospital glycoprotein (GP) IIb/IIIa inhibitors are proposed to reduce thrombus burden and improve reperfusion success in primary PCI given its rapid onset in intravenous administration. Examples include abciximab, eptifibatide and tirofiban. While they are generally associated with a greater reduction in ST-segment deviation post-PCI, data are conflicting as to whether this translates to better clinical outcomes. The FINESSE trial is the largest trial to evaluate the use of upstream GP $\mathrm{IIb} / \mathrm{III}$ in inhibition using abciximab or combination of abciximab-tenecteplase compared with PCI alone in STEMI patients intended for primary PCI [19]. In 2452 patients, there was no difference in mortality between any of the patient groups at 90 days follow-up (between 4.5 and $5.5 \%$ for all three groups; $p=0.49$ ). Conversely, in the On-TIME 2 trial, pre-PCI tirofiban was associated with fewer major adverse cardiovascular events at 30 days compared with placebo (5.8\% and $8.6 \%$, respectively; $p=0.04)$ and a trend towards reduced mortality at 1 year $(3.7 \%$ and $5.8 \%$, respectively; 0.08) [20]. Given mortality is significantly reduced in patients who receive early compared with late GP IIb/IIIa inhibitors [21], it should be noted that 
time from symptom onset to drug therapy was $165 \mathrm{~min}$ in the FINESSE trial which was much longer compared with $\sim 75 \mathrm{~min}$ in On-TIME 2. Further to the conflicting evidence for clinical benefit, these agents are highly potent and could unnecessarily increase the risk of bleeding, particularly where the diagnosis of STEMI is not definitive. In view of these limitations, current guidelines do not recommend the use of GP IIb/IIIa inhibitors in the prehospital setting [5].

\subsubsection{Fibrinolytic Therapy}

Fibrinolytic therapy was previously the mainstay treatment for STEMI patients with early presentation from symptom onset. Its rapidly waning beneficial effects with ongoing myocardial injury highlight the importance of early administration; mortality is more than halved when given less than $2 \mathrm{~h}$ from symptom onset compared with later [22]. However, more than two decades ago, primary coronary angioplasty was shown to significantly reduce death, re-infarction and hospital readmission compared with thrombolysis [23]. While subsequent findings from the CAPTIM trial suggest fibrinolysis may be equivalent if administered early in intermediaterisk STEMI patients and where rescue angioplasty is readily available [24], the further development of stent technology has since established primary PCI as the optimal reperfusion strategy for STEMI patients presenting within $12 \mathrm{~h}$ of symptom onset. However, the success of any reperfusion strategy is still dependent on total ischaemic time. Patients should not be delayed with treatment where a primary PCI facility is not within reasonable proximity, either by EMS vehicle or air transportation. Where a predefined time threshold according to local protocols cannot be met, the role of fibrinolytic therapy remains a critical aspect of prehospital STEMI management.

Fibrinolytic therapy should be administered prehospital (preferably $<10 \mathrm{~min}$ of STEMI diagnosis) and within $12 \mathrm{~h}$ of symptom onset in the absence of contraindications if a primary PCI facility is logistically unavailable. Fibrin-specific agents are preferred (e.g. tenecteplase) over systemic lytic agents (e.g. streptokinase) and should be co-administered with age-adjusted doses of dual antiplatelet and anticoagulation therapy. In the PCI-CLARITY study, adjunct clopidogrel significantly reduced the rate of major adverse cardiovascular events compared with aspirin alone in STEMI patients receiving fibrinolytic therapy (relative risk reduction, 40\%) [25]. At present, no studies have evaluated the adjunct use of ticagrelor or prasugrel with fibrinolytic therapy, and thus clopidogrel is the preferred choice in this setting. For adjunct anticoagulation, enoxaparin is the preferred treatment and is associated with fewer deaths, re-infarction or urgent revascularization compared with UFH for STEMI patients scheduled for fibrinolysis [26]. When considering fibrinolytics in the context of optimal adjunct therapy, the risk of bleeding, particularly intracranial, must be weighed against the expected benefit. Absolute contraindications include previous intracranial haemorrhage, ischaemic stroke within the last 6 months, any central nervous system malformations, gastrointestinal bleed within the last month, known bleeding disorders or possible aortic 
dissection. Certain patient characteristics such as elderly, female gender or chronic kidney disease also increase bleeding risk, and risk prediction scores are available although should not unnecessarily delay reperfusion therapy [27]. In STEMI patients with cardiogenic shock, fibrinolysis does not improve clinical outcomes possibly due to decreased coronary perfusion, and in this setting, primary PCI is recommended.

\subsubsection{Role of PCI After Fibrinolytic Therapy}

The necessity and timing of PCI after administered fibrinolytic therapy has been an area of controversy since the advent of angioplasty. Several trials have sought to evaluate the role of immediate coronary angiographic assessment (i.e. facilitated PCI) compared with conservative, ischaemia-driven angiography after fibrinolysis. The GRACIA-1 trial randomized 500 patients with thrombolysed STEMI to either facilitated PCI or conservative, ischaemia-driven management with the primary end point being death, re-infarction or revascularization at 12 months [28]. Patients in the facilitated PCI group had lower frequency of the primary end point (9\% vs. $21 \% ; p<0.001)$ : however, the time from symptom onset to fibrinolysis was $3 \mathrm{~h}$. With such delay, the optimal benefit of fibrinolysis may have passed, thus favouring PCI. The ASSENT-4 PCI trial also evaluated facilitated PCI and reported higher rates of adverse cardiovascular events (19\% vs. $13 \%$, respectively; $p=0.005)$ and stroke $(1.8 \%$ vs. $0 \%$, respectively; $p<0.0001)$ compared with PCI alone in patients with an anticipated PCI delay [29]. Routine fibrinolytic therapy with immediate PCI in patients with anticipated PCI delay could heighten the risk of intracranial bleeding in the context of adjunct DAPT and peri-procedural anticoagulants, which may in turn outweigh the benefits of thorough reperfusion.

Focus has since shifted towards a pharmaco-invasive strategy in which earlypresenting STEMI patients receive prehospital fibrinolytic therapy and transfer to a PCI-capable facility for timely, but not necessarily immediate, angiographic assessment. This approach carries the benefit of reducing early procedural risk from PCI, while enabling rapid emergency angiography (i.e. rescue PCI) in those with failed reperfusion after fibrinolysis ( $<50 \% \mathrm{ST}$-segment elevation resolution within $90 \mathrm{~min})$ [30]. The STREAM trial confirmed the safety of this strategy, in which 1892 STEMI patients within $3 \mathrm{~h}$ of symptom onset and $>1 \mathrm{~h}$ of anticipated PCI delay were randomized to either prehospital fibrinolysis and angiographic assessment within $24 \mathrm{~h}$ or primary PCI alone [31]. No difference in the primary end point of death, shock, heart failure or re-infarct at 30 days was detected, although fibrinolysis was still associated with increased risk of intracranial haemorrhage (1.6\% vs. $0.5 \%$; $p=0.03$ ). Of note, the trial protocol mandated direct EMS transfer to a primary PCI facility which was critical to showing equivalence given more than one-third of patients required rescue PCI. Taken together, there remains a distinct role for early fibrinolytic therapy in STEMI patients with anticipated PCI delay and considered risk of bleeding, although it should be followed by transfer to a PCI-capable centre for routine early angiographic assessment. 


\subsubsection{Safe Transfer to the Heart Attack Centre}

Ensuring patient safety while minimizing total ischaemic time underlies optimal transfer of patients being managed for STEMI. Following attainment of 12-lead ECG and transmission where possible, defibrillator pads should be routinely attached with continuous cardiac monitoring throughout transfer. Supplemental oxygen therapy in hypoxaemic patients (haemoglobin-oxygen saturation <90-94\%) is part of routine initial management; however, its effect above this saturation level is currently uncertain (see next section). During immediate assessment and transfer, sublingual nitroglycerin can be administered where there is ongoing chest pain although regular non-invasive blood pressure monitoring for hypotension is needed. Intravenous morphine may also be administered where pain persists. An EMS checklist and transfer report regarding patient presentation, clinical assessment findings and received treatments, including dose, time and route of administration, is common and provides clear documentation and handover to the receiving hospital team. The ability to consistently perform these tasks while monitoring the patient's clinical status is dependent on established regional STEMI protocols. The protocol should also incorporate specific key parameters for time thresholds such that EMS personnel can rapidly determine whether primary PCI is logistically achievable and to enable quantitative and regular assessment of local outcomes compared with performance benchmarks.

\subsubsection{Oxygen Therapy in Acute Myocardial Infarction}

Oxygen therapy has historically been a part of routine initial management of STEMI patients regardless of haemoglobin-oxygen saturation. This was derived from the belief that increased oxygen delivery to an ischaemic myocardium would reduce myocardial injury. However, the AVOID trial which randomized 441 STEMI patients with $\geq 94 \%$ baseline oxygen saturation to either supplemental oxygen at $8 \mathrm{~L} / \mathrm{min}$ or no oxygen found that supplemental oxygen was associated with a significant increase in peak creatine kinase [32]. Additionally, recurrent MI $(5.5 \%$ vs. $0.9 \%, p=0.006)$ and cardiac arrhythmias $(40.4 \%$ vs. $31.4 \%, p=0.05)$ at 6 months follow-up were higher in patients receiving supplemental oxygen compared with none, although the study was not powered for clinical outcomes. Subsequently, the much larger DETO2X-AMI trial found no difference with or without supplemental oxygen in patients with suspected myocardial infarction for all-cause mortality (5.0\% and 5.1\%, respectively) and rehospitalization with $\mathrm{MI}(3.8 \%$ and $3.3 \%$, respectively) [33]. Of note, less than one-half of patients in each arm had a final diagnosis of STEMI, and the lower threshold of oxygen saturation for inclusion was $90 \%$. While it remains unclear whether supplemental oxygen is harmful in patients with oxygen saturation in the normal range, the equivalent lack of benefit suggests that it should be reserved for patients with lower saturation $(<90-94 \%)$ and we await further randomized data to elucidate this controversy. 


\subsection{Summary}

Patient outcomes following myocardial infarction are heavily dependent on the prehospital phase. Prompt recognition and correct triage of patients suffering from chest pain are paramount, with healthcare networks continually evolving to deliver upfront pharmacological treatment and rapid access to therapies that allow for myocardial reperfusion. As we move into the future, our focus as healthcare providers should remain on reducing the overall duration of myocardial ischaemia while also considering expansion of rapid reperfusion to patients with an acute coronary syndrome not currently served by existing pathways.

\section{References}

1. Gersh BJ, Stone GW, White HD, Holmes DR Jr. Pharmacological facilitation of primary percutaneous coronary intervention for acute myocardial infarction: is the slope of the curve the shape of the future? JAMA. 2005;293:979-86.

2. De Luca G, Suryapranata H, Ottervanger JP, Antman EM. Time delay to treatment and mortality in primary angioplasty for acute myocardial infarction: every minute of delay counts. Circulation. 2004;109:1223-5.

3. Goldberg RJ, Spencer FA, Fox KA, et al. Prehospital delay in patients with acute coronary syndromes (from the global registry of acute coronary events [GRACE]). Am J Cardiol. 2009;103:598-603.

4. Sorensen JT, Terkelsen CJ, Norgaard BL, et al. Urban and rural implementation of pre-hospital diagnosis and direct referral for primary percutaneous coronary intervention in patients with acute ST-elevation myocardial infarction. Eur Heart J. 2011;32:430-6.

5. Ibanez B, James S, Agewall S, et al. ESC guidelines for the management of acute myocardial infarction in patients presenting with ST-segment elevation: the task force for the management of acute myocardial infarction in patients presenting with ST-segment elevation of the European Society of Cardiology (ESC). Eur Heart J. 2017;33(20):2569-619.

6. Thygesen K, Alpert JS, Jaffe AS, et al. Third universal definition of myocardial infarction. Circulation. 2012;126:2020-35.

7. Brown AJ, Hoole SP, McCormick LM, et al. Left bundle branch block with acute thrombotic occlusion is associated with increased myocardial jeopardy score and poor clinical outcomes in primary percutaneous coronary intervention activations. Heart. 2013;99:774-8.

8. Katritsis DG, Siontis GC, Kastrati A, et al. Optimal timing of coronary angiography and potential intervention in non-ST-elevation acute coronary syndromes. Eur Heart J. 2011;32:32-40.

9. Roffi M, Patrono C, Collet JP, et al. 2015 ESC guidelines for the management of acute coronary syndromes in patients presenting without persistent ST-segment elevation: task force for the Management of Acute Coronary Syndromes in patients presenting without persistent ST-segment elevation of the European Society of Cardiology (ESC). Eur Heart J. 2016;37:267-315.

10. Mauri L, Kereiakes DJ, Yeh RW, et al. Twelve or 30 months of dual antiplatelet therapy after drug-eluting stents. N Engl J Med. 2014;371:2155-66.

11. Montalescot G, van 't Hof AW, Lapostolle F, et al. Prehospital ticagrelor in ST-segment elevation myocardial infarction. N Engl J Med. 2014;371:1016-27.

12. Wiviott SD, Braunwald E, McCabe $\mathrm{CH}$, et al. Prasugrel versus Clopidogrel in patients with acute coronary syndromes. N Engl J Med. 2007;357:2001-15.

13. Wallentin L, Becker RC, Budaj A, et al. Ticagrelor versus Clopidogrel in patients with acute coronary syndromes. N Engl J Med. 2009;361:1045-57. 
14. Zeymer U, Mochmann HC, Mark B, et al. Double-blind, randomized, prospective comparison of loading doses of $600 \mathrm{mg}$ clopidogrel versus $60 \mathrm{mg}$ prasugrel in patients with acute ST-segment elevation myocardial infarction scheduled for primary percutaneous intervention: the ETAMI trial (early thienopyridine treatment to improve primary PCI in patients with acute myocardial infarction). JACC Cardiovasc Interv. 2015;8:147-54.

15. Bhatt DL, Stone GW, Mahaffey KW, et al. Effect of platelet inhibition with Cangrelor during PCI on ischemic events. N Engl J Med. 2013;368:1303-13.

16. Montalescot G, Zeymer U, Silvain J, et al. Intravenous enoxaparin or unfractionated heparin in primary percutaneous coronary intervention for ST-elevation myocardial infarction: the international randomised open-label ATOLL trial. Lancet. 2011;378:693-703.

17. Valgimigli M, Frigoli E, Leonardi S, et al. Bivalirudin or unfractionated heparin in acute coronary syndromes. N Engl J Med. 2015;373:997-1009.

18. Yusuf S, Mehta SR, Chrolavicius S, et al. Effects of fondaparinux on mortality and reinfarction in patients with acute ST-segment elevation myocardial infarction: the OASIS-6 randomized trial. JAMA. 2006;295:1519-30.

19. Ellis SG, Tendera M, de Belder MA, et al. Facilitated PCI in patients with ST-elevation myocardial infarction. N Engl J Med. 2008;358:2205-17.

20. ten Berg JM, van 't Hof AW, Dill T, et al. Effect of early, pre-hospital initiation of high bolus dose tirofiban in patients with ST-segment elevation myocardial infarction on short- and longterm clinical outcome. J Am Coll Cardiol. 2010;55:2446-55.

21. DEL G, Bellandi F, Huber K, et al. Early glycoprotein IIb-IIIa inhibitors in primary angioplasty-abciximab long-term results (EGYPT-ALT) cooperation: individual patient's data meta-analysis. J Thromb Haemost. 2011;9:2361-70.

22. Boersma E, Maas AC, Deckers JW, Simoons ML. Early thrombolytic treatment in acute myocardial infarction: reappraisal of the golden hour. Lancet. 1996;348:771-5.

23. Investigators. TGUoStOOCAiACSAS. A clinical trial comparing primary coronary angioplasty with tissue plasminogen activator for acute myocardial infarction. $\mathrm{N}$ Engl J Med. 1997;336:1621-8.

24. Bonnefoy E, Lapostolle F, Leizorovicz A, et al. Primary angioplasty versus prehospital fibrinolysis in acute myocardial infarction: a randomised study. Lancet. 2002;360:825-9.

25. Sabatine MS, Cannon CP, Gibson CM, et al. Effect of clopidogrel pretreatment before percutaneous coronary intervention in patients with ST-elevation myocardial infarction treated with fibrinolytics: the PCI-CLARITY study. JAMA. 2005;294:1224-32.

26. Antman EM, Morrow DA, McCabe CH, et al. Enoxaparin versus unfractionated heparin with fibrinolysis for ST-elevation myocardial infarction. N Engl J Med. 2006;354:1477-88.

27. Moscucci M, Fox KA, Cannon CP, et al. Predictors of major bleeding in acute coronary syndromes: the global registry of acute coronary events (GRACE). Eur Heart J. 2003;24:1815-23.

28. Fernandez-Aviles F, Alonso JJ, Castro-Beiras A, et al. Routine invasive strategy within 24 hours of thrombolysis versus ischaemia-guided conservative approach for acute myocardial infarction with ST-segment elevation (GRACIA-1): a randomised controlled trial. Lancet. 2004;364:1045-53.

29. Assessment of the Safety and Efficacy of a New Treatment Strategy with Percutaneous Coronary Intervention (ASSENT-4 PCI) investigators. Primary versus tenecteplase-facilitated percutaneous coronary intervention in patients with ST-segment elevation acute myocardial infarction (ASSENT-4 PCI): randomised trial. Lancet. 2006;367:569-78.

30. Gershlick AH, Stephens-Lloyd A, Hughes S, et al. Rescue angioplasty after failed thrombolytic therapy for acute myocardial infarction. N Engl J Med. 2005;353:2758-68.

31. Armstrong PW, Gershlick AH, Goldstein P, et al. Fibrinolysis or primary PCI in ST-segment elevation myocardial infarction. N Engl J Med. 2013;368:1379-87.

32. Stub D, Smith K, Bernard S, Nehme Z, Stephenson M, Bray JE, Cameron P, Barger B, Ellims AH, Taylor AJ, Meredith IT, Kaye DM, AVOID Investigators. Air vs. oxygen in ST-segmentelevation myocardial infarction. Circulation. 2015;131(24):2143-50.

33. Hofmann R, James SK, Jernberg T, et al. Oxygen therapy in suspected acute myocardial infarction. N Engl J Med. 2017;377:1240-9. 
Open Access This chapter is licensed under the terms of the Creative Commons Attribution 4.0 International License (http://creativecommons.org/licenses/by/4.0/), which permits use, sharing, adaptation, distribution and reproduction in any medium or format, as long as you give appropriate credit to the original author(s) and the source, provide a link to the Creative Commons license and indicate if changes were made.

The images or other third party material in this chapter are included in the chapter's Creative Commons license, unless indicated otherwise in a credit line to the material. If material is not included in the chapter's Creative Commons license and your intended use is not permitted by statutory regulation or exceeds the permitted use, you will need to obtain permission directly from the copyright holder. 